\title{
PERFORMANCE IMPROVEMENT OF BOTTLENECK LINK IN RED VEGAS OVER HETEROGENEOUS NETWORKS
}

\author{
S. P. Angelin Claret \\ Asst.Prof, Dept. of Computer Applications, SRM University, Tamilnadu, India, angelin.s@ktr.srmuniv.ac.in
}

\begin{abstract}
In communication networks, congestion avoidance in routers is one of the hottest topics. Current IP network has become the dominant paradigm for all networking environments. The significant cause of packet losses in such heterogeneous networks is no longer limited to network congestion. Traditional TCP interprets every packet loss as caused by congestion which may be not the case in the current Internet. TCP Vegas detects network congestion in the early stage and successfully prevents periodic packet loss that usually occurs in TCP Reno. In this paper a new variant of TCP Vegas namedRed Vegas has used. The Red Vegas may detect random packet losses precisely. Through the packet loss differentiation, Red Vegas reacts appropriately to the losses, and therefore the throughput of connection over heterogeneous networks significantly improved. The bandwidth of the bottleneck link is under-utilized when the random loss rate is high. Therefore, a new design of the fast recovery mechanism for Red Vegas would be carried out. The proposed mechanism improves the aggression of Vegas TCP in competing for the available bandwidth whilst maintaining the inherent stability of the original Vegas TCP scheme.
\end{abstract}

Index Terms: TCP Vegas, RED Vegas, Bandwidth and Congestion Control

$* * * * *$

\section{INTRODUCTION}

Internet congestion occurs when the aggregate demand for a resource (e.g., link bandwidth) exceeds the available capacity of the resource. Resulting effects from such congestion include long delays in data delivery, wasted resources due to lost or dropped packets and even possible congestion collapse, in which all communication in the entire network ceases. It is therefore clear that in order to maintain good network performance; certain mechanisms must be provided to prevent the network from being congested for any significant period of time. Two approaches to handling congestion are congestion control (or recovery) and congestion avoidance. The former is reactive in that congestion control typically comes into play afterthe network is overloaded, i.e., congestion is detected. The latter is proactive in that congestion avoidance comes into play beforethe network becomes overloaded, i.e., when congestion is expected. Compared with fixed and wired networks, the wireless networks have high bit-error rate (BER) and offer less available bandwidth. Furthermore, the host mobility also results in packet lossor delay during handoff process. TCP responds to all losses by invoking congestion control and avoidance algorithms, resulting in the degraded end-to-end performance unnecessarily.

\section{THE PRIMARY FACTORS THAT AFFECT WIRELESS TCP PERFORMANCE}

\subsection{Limited Bandwidth}

The rate of the wired LANS is IOOMbps and the optical fiber link may provide the rate exceeding IGbps, but the rate of thewireless LANS regulated in the IEEE 802.11 is only 11 Mhps. obviously, the limited bandwidth will become the bottleneck to enhance wireless TCP performance.

\subsection{Long Round Trip Times}

The latencies of the wireless medium are much longer than those of the wired medium and the rate to increase thecongestion window sizes of the sender is in linear growth with the rate that it receives ACKS. Thus, because of the long R'IT, the growth rate of the congestion window in the wireless link will be very low, and it will reduce the wireless TCP throughput.

\subsection{Random Losses}

There are often bursts of errors due to poorsignal strength in an area or duration of noise. It is common that sequential packet losses emerge due to the bursts of errors, but each packet loss can cause the congestion window to reduce half, and then the sending rate reduces seriously. 


\subsection{User Mobility}

When a mobile transfers from one cell to another, mobile handoff will occur. In the handoff process, all essential information must be transmitted between the two base stations, and then mobile hosts are able to maintain the connection. The handoff process usually takes about $300 \mathrm{~ms}$, so some packets may loss during this period of time.

\subsection{Short Flows}

The majority of the data in the wireless networks transmission belong to the short flows service. At the beginning of the connection, TCP is still in the state of slow-start. The data transmission maybe has been already completed before the widow of the sender reaches its maximum, and it cannot make full use of the link.

\subsection{Power Consumption}

The power consumption plays a dominant role in determining battery life, so it highly affects the wireless equipment, such as laptop computer, PDA, radiotelephone and so on. The retransmission caused by the frequent packet losses makes TCP connection time quite long, therefore power consumption is quite high.

\section{OPEN ISSUES}

There still persist many open issues related to the design, operation, and evaluation of the Internet congestion control mechanisms. The major open issues are interoperability, robustness, stability, convergence, implementation complexity, and fairness. Since congestion occurs when the network is overloaded and is closely related to the network traffic environments, there are related open issues that should be taken into account for solving congestion control. These related issues are link characteristics, TCP-friendliness vs. user datagram protocol (UDP) traffics, and assumptions on network traffic dynamics.

\subsection{Interoperability and Robustness}

Floyd et alnote that an increasing deployment of traffic lacking end-to-end congestion control may cause congested links to occupy themselves sending packets that will only be dropped later in the network. They describe how this leads to congestion collapsein the network. Therefore, the heterogeneity of congestion control mechanisms and the lack of end-to-end congestion control may result in an interoperability problem. This problem may also cause inefficient performance when all the different algorithms have conflicting optimization goals such as power and (net) benefit, etc. It is also necessary to maintain TCP's robust nature in the presence of packet losses and a wide range of offered load, link speeds, packet sizes, and congestion levels. A robust algorithm will be insensitive to the various traffic environments.

\subsection{Stability, Convergence and Implementation Complexity}

Two concepts of stability exist in Internet congestion control. First, Internet congestion control may have asymptotic convergence to a fixed operating point. Second, it may have controlled oscillation points, i.e., limited cycles with bounded variations. In both cases, the objective of a congestion control algorithm is to achieve steady-state performance in terms of link utilization, throughput, and RTT.

\subsection{Fairness in Internet Congestion Control}

Two fairness issues may occur in Internet congestion control: The fair bandwidth sharing between competing connections and the fair marking(or dropping) of packets when congestion occurs. The fair sharing of bandwidth among connections depends on the fact that all connections are running basically the same congestion avoidance algorithms, conforming to the current TCP specification. However, fair marking has not been studied actively. The issue of fair bandwidth sharing among competing TCP connections has become increasingly important for several reasons. One reason is due to the growth of individual TCPs that can use high bandwidth even over high-propagation-delay paths. Second, with the growth of the Web, Internet users want high-bandwidth and low-delay communications.

\subsection{Fairness in Bandwidth Allocation}

A bandwidth allocation scheme is fairif it does not offer different treatment to connections, either based on the time order in which they request a share of the available bandwidth, or on the particular location of their source and destination points. A set of rates is max-min fairif no rate may be increased without simultaneously decreasing another rate which is already smaller. In a network with a single congested link, max-min fairness implies an equal share of the bandwidth for each connection through it. To achieve maxmin fairness, the global optimality of the network, often called Pareto efficiency, needs to be sacrificed.

\section{HETEROGENEOUS NETWORK}

It is a network connecting computers and other devices. Different operating systems and/or protocols.

\section{RANDOM EARLY DETECTION (RED)}

Also known as random early discard or random early drop.It uses an active queue management algorithm and a congestion avoidance algorithm

\subsection{How RED Works}

RED monitors the average queue size. It Drops packets based on statistical probabilities. 


\subsubsection{Statistical Probabilities}

Buffer---->empty, all incoming packets are accepted.

Buffer----> full,

all incoming packets are dropped.

\section{COMPARISON BETWEEN VEGAS AND RENO}

$>$ Detects network congestion in the early stage.

$>$ Successfully prevents periodic packet loss.

$>$ The Red Vegas may detect random packet losses precisely.

$>$ Red Vegas reacts appropriately to the losses.

$>$ The throughput of connection over heterogeneous networks is significantly improved

$>$ TCP Vegas host-dominated configurations perform better than TCP Reno host-dominated configurations.

\subsection{Congestion window size variation in Reno and}

Vegas

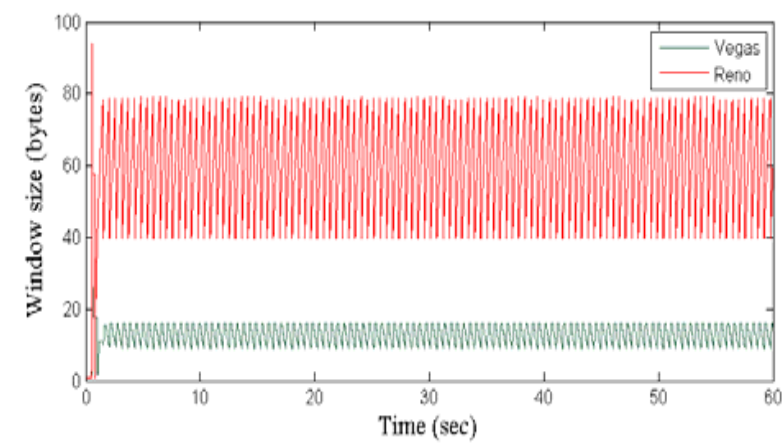

Fig: 1 Interaction of TCP in Heterogeneous Network

\subsection{Congestion window size variation in Reno and} modified Vegas

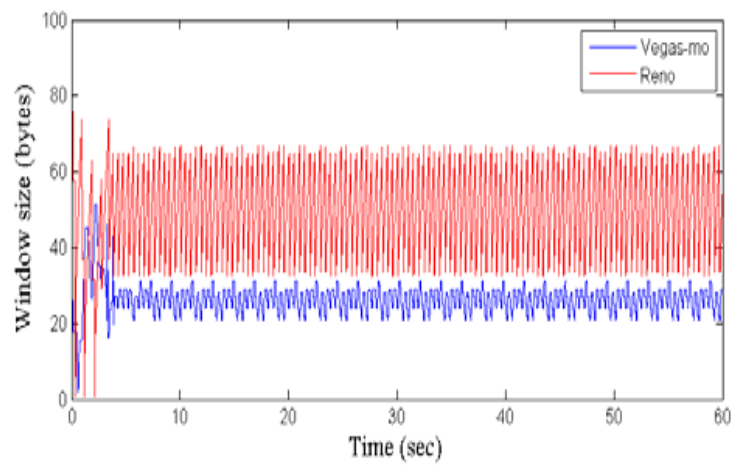

Fig:2 Interaction of TCP in Heterogeneous Network (With Link-Level Bit Error)

\section{1 .1 TCP flavors Goodput (Mbps)}

$>$ Reno/Vegas 23.59/16.53

$>$ Reno/Vegas-mo 22.14/17.81

\subsubsection{Performance Analysis for RED}

$>$ The RED improvement is higher (mix of Reno and Vegas hosts).

$>$ RED algorithm provides better fairness

\subsubsection{Why RED in Vegas}

$>$ Vegas adopts a more sophisticated bandwidth estimation

$>$ Controls the congestion window size accordingly.

\section{BANDWIDTH}

$>$ The bandwidth of the bottleneck link is underutilized when the random loss rate is high.

$>$ Therefore, a new design of the fast recovery mechanism for Red Vegas would be carried out.

\section{CONCLUSIONS}

This paper discussed a few issues of TCP Vegas. TCP Vegas could cause a strange behavior with RED Vegas when there is a rerouting in the network and Performance Improvement of bottleneck link in RED Vegas over Heterogeneous Networks connections. Also shown that TCP Vegas could lead the network to a persistent congestion if connections start at various times This problem could be solved using the combination of the RED gateways and the same modification proposed for the problem of rerouting. This brings about a more even distribution of the bandwidth regardless of the starting time and guarantees that the congestion level stays around the desired congestion level by the RED gateways. Finding the appropriate threshold values for the RED gateways, however, is still an open problem. If the threshold values are set too low, it may cause too many packet drops initially before the connections settle or the window sizes may never settle, which is undesirable.

\section{REFERENCES}

[1] V. Jacobson, "Congestion Avoidance and Control," Proc. ACM SIGCOMM '88, 1988, pp. 314-29.

[2] S. Floyd and K. Fall, "Promoting the Use of the End-toEnd Congestion Control in the Internet," IEEE/ACM Trans. Net., vol. 7, no. 4, Aug. 1999, pp. 458-72.

[3] S. Floyd, "Internet Research: Comments on Formulating the Problem," unpublished manuscript, http://www.aciri.org/floyd/, Jan. 1998.

[4] S. Floyd, "Congestion Control Principles," IETF draft, drafts Floyd- cong-01.txt, Jan. 2000. 
[5] D. Wischik, "How to Mark Fairly," http://www.statslab.cam.ac. UK/ djw1005/, Sept. 1999.

[6] M. Allman, V. Paxson, and W. Stevens, "TCP Congestion Control," IETF RFC2581, Apr. 1999.

[7] F. P. Kelly, A. K. Maulloo, and D. K. H. Tan, "Rate Control in Communication Networks: Shadow Prices, Proportional Fairness, and Stability," J. Operational Research Society, vol. 49, http://www.statslab.cam.ac.uk/ frank/rate.html, pp. 237-52.

[8] D. P. Bertsekas and R. G. Gallager, Data Networks, Prentice- Hall, 2nd Ed., 1992.

[9] F. Bonomi and K. W. Fendick, "The Rate-Based Flow Control Framework for the Available Bit Rate ATM Service," IEEE Network, vol. 9, no. 2, Mar. /Apr. 1995, pp. 25-39.

[10] S. Floyd and V. Jacobson, IRandom Early Detection Gateways for Congestion Avoidance",

IEEE/ACM Transactions on Networking, Vol. 1, No, 4, pp. 397-413, August 1993. 